\title{
Association of paraoxonase gene polymorphisms with diabetic nephropathy and retinopathy
}

\author{
JUN WANG $^{1 *}$, MING MING YANG ${ }^{2,3 *}$, SHI SONG RONG ${ }^{3}$, TSZ KIN NG $^{4}$, YAN BO LI ${ }^{1}$ and XIAO MIN LIU \\ ${ }^{1}$ Department of Endocrinology and ${ }^{2}$ Eye Hospital, The First Affiliated Hospital of Harbin Medical University, \\ Harbin, Heilongjiang 150001; ${ }^{3}$ Department of Ophthalmology and Visual Sciences, The Chinese University \\ of Hong Kong, Hong Kong SAR, P.R. China; ${ }^{4}$ Geriatric Research, Education and Clinical Center, \\ Miami Veterans Affairs Medical Center, Miami, FL 33125, USA
}

Received April 24, 2013; Accepted September 26, 2013

DOI: $10.3892 / \mathrm{mmr} .2013 .1710$

\begin{abstract}
Emerging reports have revealed a potential association of paraoxonase $(P O N)$ gene polymorphisms with diabetic nephropathy (DN) and diabetic retinopathy (DR). However, the identification of susceptible genes and the quantification of associated risks are elusive owing to a lack of reproducibility. Therefore, a meta-analysis was conducted in the present study to improve the understanding of the effect of PON1 and PON2 on DN and DR. A total of 10 articles, involving 2,877 patients and 3,246 controls met the inclusion criteria. Functional variants $(n=4)$ were evaluated, including rs662 (p.Q192R) and rs854560 (p.L55M) in PON1; and rs7493 (p.S311C) and rs12026 (p.A148G) in PON2. Overall, PON1-L55M was found to be significantly associated with DR in all the genetic models: allele [odds ratio $(\mathrm{OR})=2.42 ; 95 \%$ confidence interval (CI), 1.91-3.07]; dominant (OR=5.76; 95\% CI, 3.14-10.55), homozygote $(\mathrm{OR}=10.53 ; 95 \% \mathrm{CI}, 5.59-19.86)$, heterozygote $(\mathrm{OR}=3.62 ; 95 \% \mathrm{CI}, 1.94-6.74)$, and recessive $(\mathrm{OR}=3.56$; $95 \% \mathrm{CI}$, 2.61-4.86), with no evidence of between-study heterogeneity. However, such associations were not detected in DN and the other three polymorphisms did not show any associations with DN or DR. The current meta-analysis highlighted results for the risk of association of $P O N 1-55 \mathrm{~L}$ with DR. The results also indicated that PON2 gene polymorphisms, as well as PON1-Q192R, may not confer major genetic risk to DN or DR. Additional studies are required to enrich the understanding of $P O N$ genes, particularly for its functional role in DR.
\end{abstract}

\section{Introduction}

The prevalence of diabetes is reaching epidemic proportions at an alarming rate worldwide. Diabetes mellitus (DM) is a group

Correspondence to: Professor Xiao Min Liu, Department of Endocrinology, The First Affiliated Hospital of Harbin Medical University, 23 Post Road, Harbin, Heilongjiang 150001, P.R. China E-mail: liuxiaomin_1957@163.com

*Contributed equally

Key words: paraoxonase, diabetic nephropathy, diabetic retinopathy of metabolic diseases induced by insulin secretion deficiency and/or insulin resistance, which result in chronic hyperglycemia. Diabetic nephropathy (DN) and diabetic retinopathy (DR), as major microvascular complications, are the leading cause of end-stage renal disease and loss of vision, respectively (1). It is well known that prolonged hyperglycemia is an important risk factor $(2,3)$. An important conceptual consideration is that the diseases manifest in individuals with genetic predisposition coupled with environmental triggers $(4,5)$. Previous studies (6-8) have focused on the genetic basis of diabetes and its complications by highlighting methods for improving and understanding the mechanisms involved in the disease.

Lipoprotein oxidation was previously found to be involved in the development of cerebrovascular and coronary artery diseases, as well as microvascular complications of diabetes (9-11). Paraoxonase $(P O N)$ is a high-density lipoprotein (HDL)-associated enzyme, protecting lipoproteins from oxidation (12). The PON gene clusters (PON1 and PON2) mapped on human chromosome $7 \mathrm{q} 21.3$ with several polymorphisms, particularly specific functional variants with possible biological effects on enzyme activity, have been extensively evaluated as genetic candidates for diabetic microvascular complications. These include the following: PON1 rs662 (c.575A $>$ G or p.Gln192Arg or p.Q192R), PON1 rs854560 (c.163T >A or p.Leu55Met or p.L55M), PON2 rs7493 (c.932C $>$ G or p.Ser311Cys or p.S311C) and PON2 rs12026 (c.443C $>$ G or p.Ala148Gly or p.A148G) $(6-8,13-19)$. These emerging observations in genetic predisposition to DN and DR have drawn particular attention and therefore, have garnered research interests. The correlation between $P O N$ polymorphisms and disorders, as aforementioned, remain unclear since the reproducibility of a number of initial associations have not been forthcoming and specific results from small sample sizes are often controversial. Therefore, a meta-analysis was conducted in the present study to mitigate these shortcomings and evaluate the genetic effects of PON1 and PON2 genes on the risk of DN and DR.

\section{Materials and methods}

Search strategy and inclusion criteria. Online databases, MEDLINE (Medical Literature Analysis and Retrieval 
System Online) and EMBASE (via Ovid) were used for the literature search between the starting dates of the databases and 6 January, 2013. The keywords were used as free words and also as MeSH terms: 'paraxonase', 'PON1', 'PON2', 'diabet(es/ic)', 'nephropathy(ies)', 'retinopathy(ies)', 'microvascular complication (s)', 'polymorphism(s)', 'variant(s)' and 'mutation(s)'. Reference lists of the retrieved articles and reviews were also screened for additional articles not obtained by the electronic search.

The inclusion criteria were defined as follows: i) original case-control studies evaluating the association between DN/DR and $P O N$ polymorphisms; ii) numbers or frequencies in case and control groups reported for each genotype or allele; iii) study samples of unrelated individuals drawn from clearly defined populations; and iv) studies using diabetic patients free from any form of complications as the control group. Animal studies, case reports, reviews, abstracts, conference proceedings, editorials, reports with incomplete data and studies based on pedigree data were excluded.

Literature review and data extraction. All the articles retrieved were reviewed and data extracted by two independent investigators with standardized datasheets. Uncertainties were resolved by consensus with a third reviewer. Information collected from each study included: first author, year of publication, country of study, ethnicity, diagnostic methods of DN and DR, sample size, polymorphisms studied and allelic and genotypic frequencies.

If genotype or allele data were not available in the publication, calculations were based on the tests for Hardy-Weinberg equilibrium (HWE) in the original study. If the test for HWE was not reported, it was tested by genotype data.

Statistical analysis. HWE was evaluated using the $\chi^{2}$ test. Pooled odds ratios (ORs) and 95\% confidence intervals (CIs) were estimated with the DerSimonian and Laird random-effects model. Although random-effects analyses exhibited less power than analyses based on fixed-effects models, they yielded a more conservative CI (20). For the genotypic comparison, dominant, homozygote, heterozygote and recessive models were applied into the investigation of the disease association with reference to the common variation (Q for p.Q192R; L for p.L55M; S for p.S311C; and A for p.A148G). Cochran's Q statistic was used to test heterogeneity across studies and the index $I^{2}$ statistic was used to quantify the proportion of total variation attributable to between-study heterogeneity. $\mathrm{P}<0.1$ was considered to indicate a statistically significant difference for Q-statistic and $I^{2}>50 \%$ was considered to indicate large heterogeneity. The sensitivity analysis was applied to assess the stability of the results. Funnel plot asymmetry and modified Egger's regression test were used to statistically assess the potential bias. Data management and statistical analyses were conducted with 'metafor' package v1.6-0 and 'Hardy Weinberg' package v1.3 in R language v2.15.0. $\alpha$ was set to 0.05 .

\section{Results}

Study identification and characteristics. Major bibliographic databases were screened, searching for studies focusing on

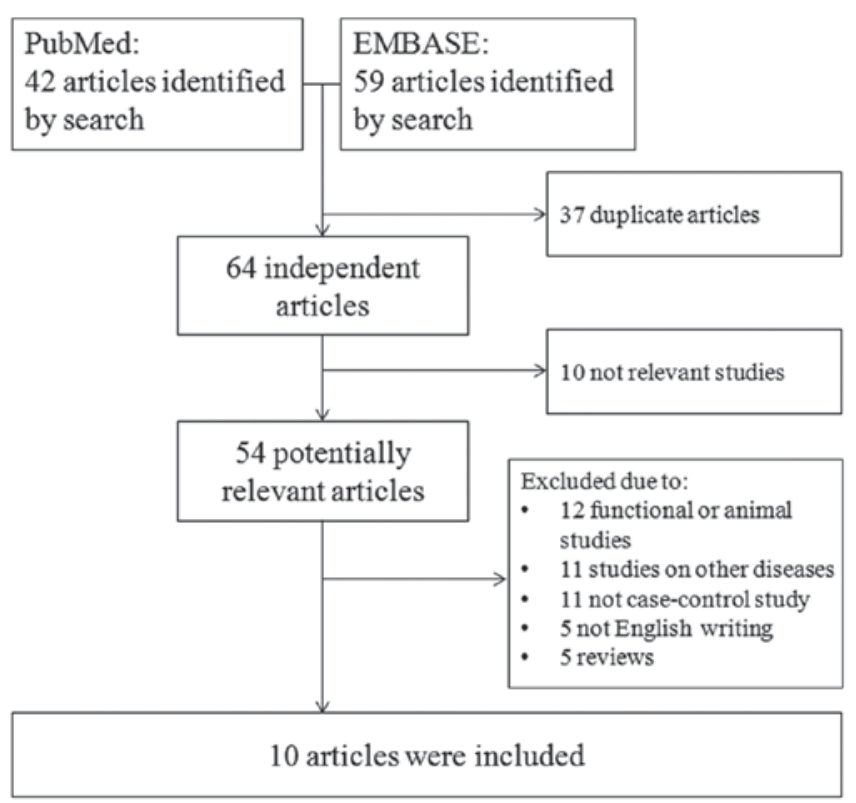

Figure 1. A schematic representation of the search strategy and selection process.

the associations of $P O N 1$ and $P O N 2$ polymorphisms with DR and DN. A schematic representation of the selection process with specific reasons is presented in Fig. 1. The initial search strategy retrieved 101 potentially relevant studies. Following screening, a total of 10 studies with 23 outcomes met the inclusion criteria used for the meta-analysis. General characteristics and genotypic frequencies of these reports are presented in Table I. Overall estimates of $P O N$ gene (PON1 and PON2) polymorphisms for DN and DR in dominant model are shown in Fig. 2.

Meta-analysis of PON1-Q192R. The association of Q192R with DN was assessed in five studies. Of these, two studies were performed in Caucasian populations and three in Asian populations. All the control groups were in HWE, with the exception of the study by Ergun et al (14). A random-effects model that takes into account the intra- and inter-study variability did not reveal any significant association of Q192R with $\mathrm{DN}$, under any of the following genetic models: allele $(\mathrm{Q}$ vs., $\mathrm{R}$ : $\mathrm{OR}=0.90 ; 95 \% \mathrm{CI}, 0.73-1.11)$, dominant $(\mathrm{QQ}+\mathrm{QR}$ vs., RR: $\mathrm{OR}=0.84$; 95\% CI, 0.49-1.15), homozygote (QQ vs., RR: $\mathrm{OR}=0.79 ; 95 \% \mathrm{CI}, 0.41-1.53)$, heterozygote (QR vs., RR: $\mathrm{OR}=0.84 ; 95 \% \mathrm{CI}, 0.44-1.60)$, and recessive (QQ vs., $\mathrm{QR}+\mathrm{RR}$ : $\mathrm{OR}=0.91$; 95\% CI, 0.64-1.31) (Table II). These ORs were moderately heterogeneous across studies in overall comparisons. The frequency of the Q allele in Indian populations was found to be the major allele, similar to that of Caucasian populations, while the $\mathrm{Q}$ allele was found to be the minor allele in the single Japanese population. When stratifying by ethnicity, no evident associations were found in the Caucasian or Asian populations. Publication bias was assessed using funnel plots, which indicated symmetry of the genetic effects for these ORs (data not shown).

To assess the association of Q192R with DR, three studies were conducted. No significant associations were found in the genetic models when the studies were pooled into the 


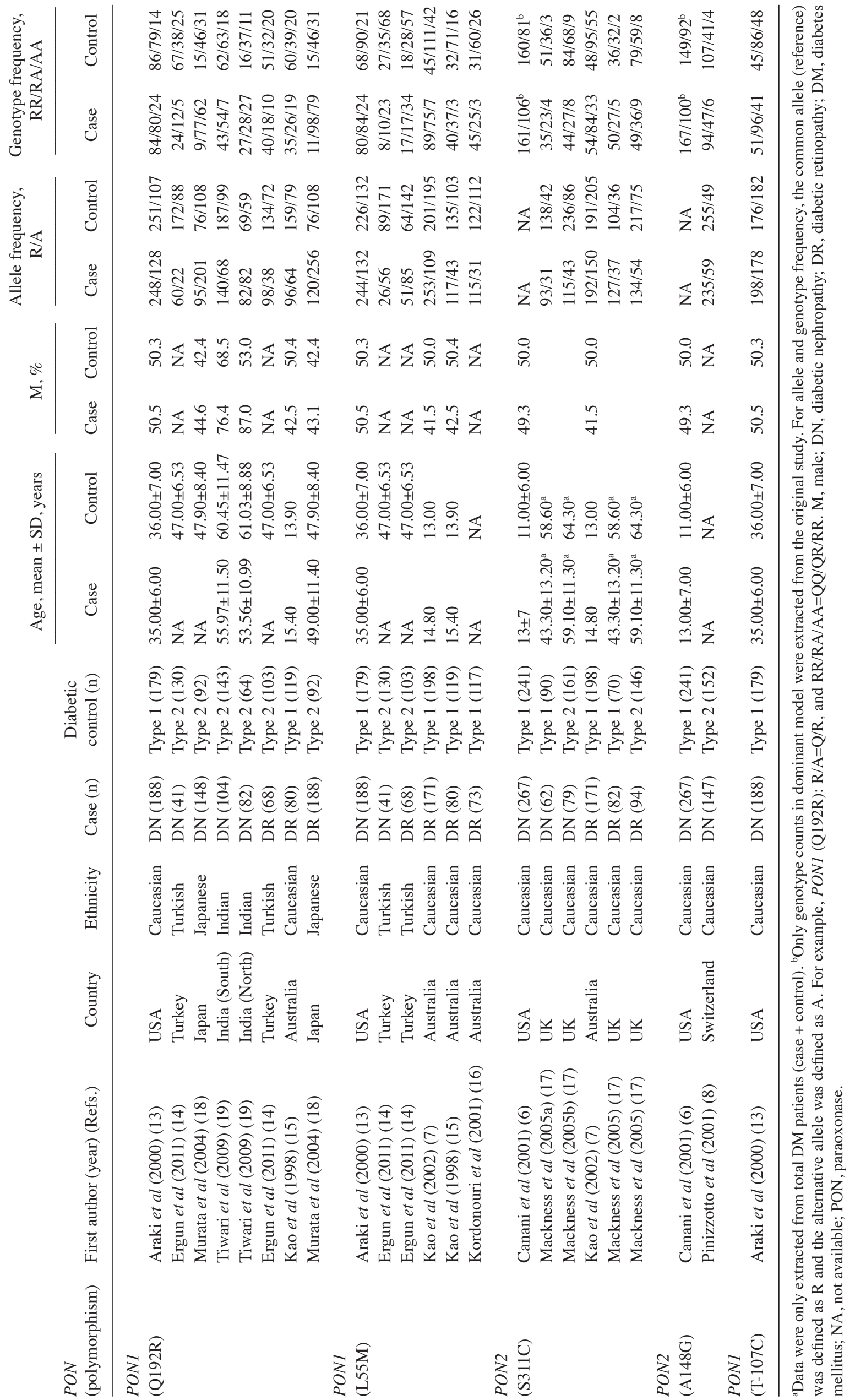


A

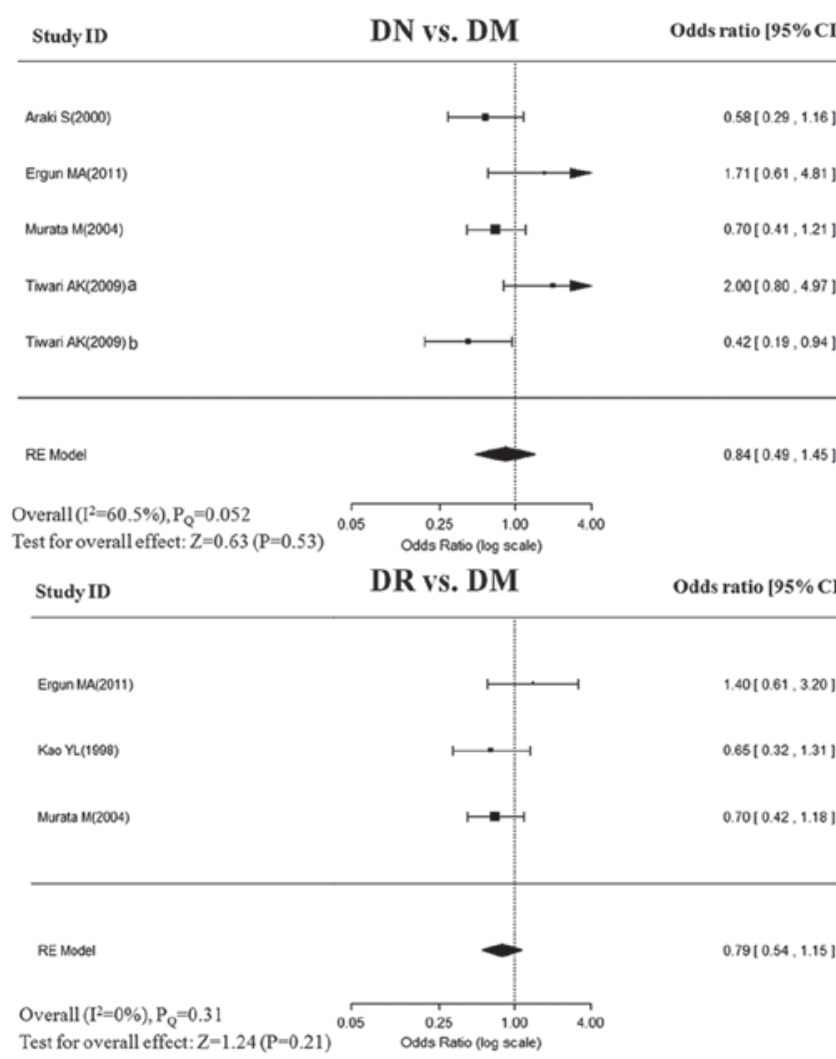

C

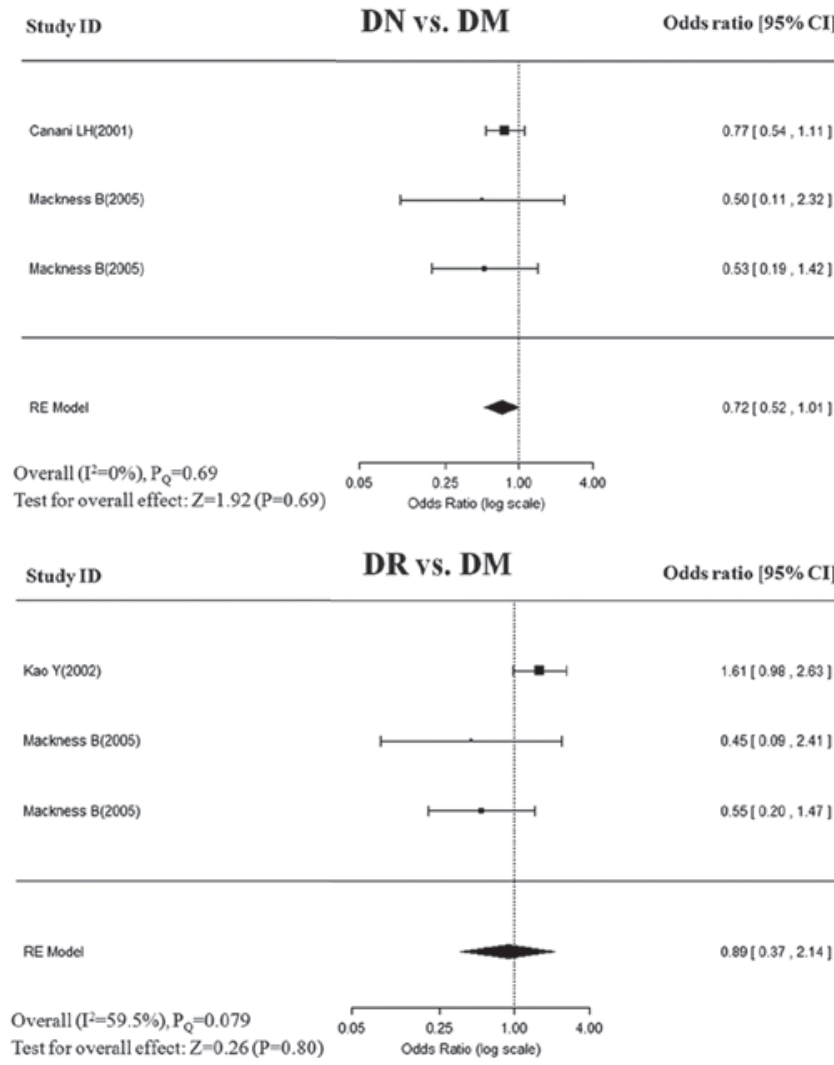

meta-analysis (Table II). In addition, no evidence of publication bias was observed. Subgroup analysis by ethnicity was not performed due to a small number of studies.

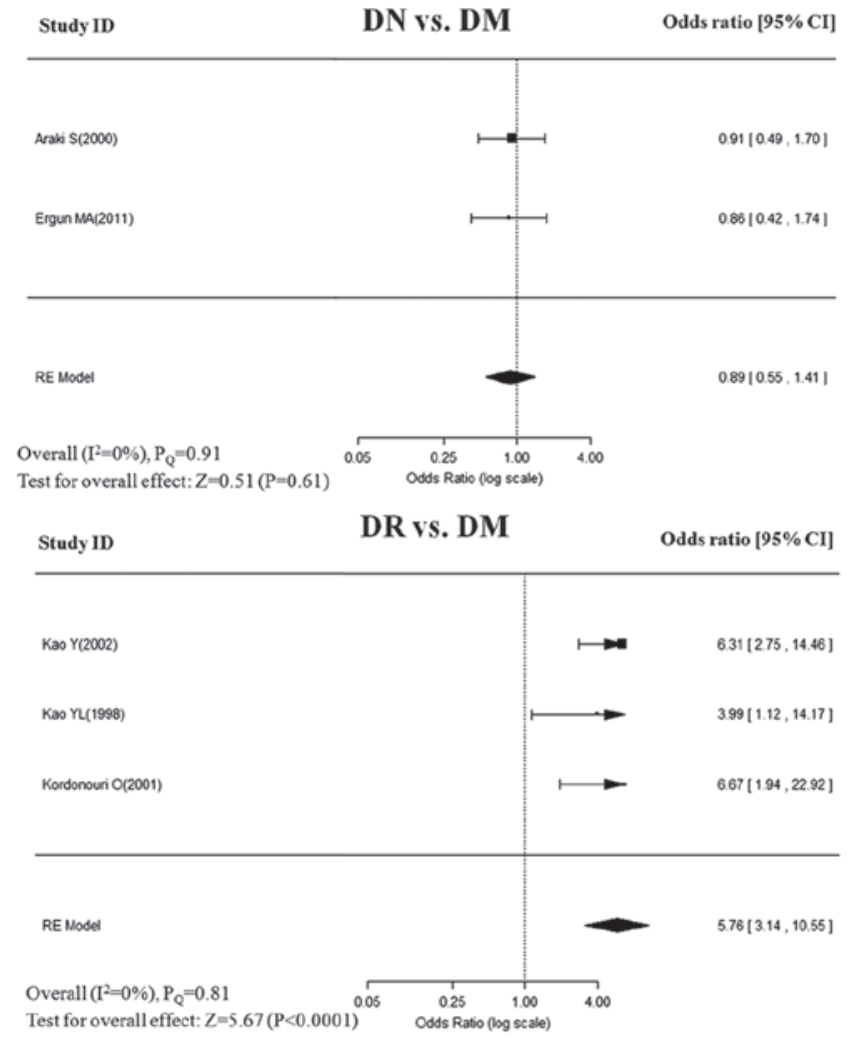

D

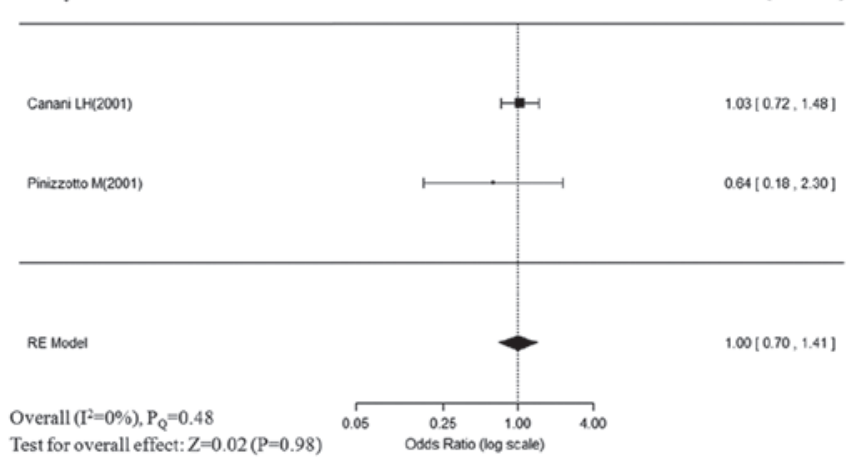

Figure 2. Overall estimates of $P O N$ gene (PON1 and $P O N 2$ ) polymorphisms for DN and DR in dominant model. The size of the box is proportional to the weight of the study, horizontal lines indicate $95 \% \mathrm{CI}$ and a diamond indicates the summary OR with its corresponding 95\% CI. (A) PON1-Q192R, (B) PON1-L55M, (C) PON2-S311C, and (D) PON2-A148G. PON, paraoxonase; DN, diabetic nephropathy; DR, diabetic retinopathy; DM, diabetes mellitus; OR, odds ratio; CI, confidence interval.

Meta-analysis of PON1-L55M. The association of L55M with risk of DN in Caucasian populations was assessed in two studies. The pooled analysis showed that no significant 
Table II. Pooled analyses on the correlation between PON gene polymorphisms and DN and DR.

\begin{tabular}{|c|c|c|c|c|c|c|c|}
\hline $\begin{array}{l}P O N \\
\text { (polymorphism) }\end{array}$ & Disease & $\begin{array}{l}\text { Sample size, } \\
\text { cases/controls }\end{array}$ & Genetic model & OR $(95 \%$ CI $)$ & P-value & $I^{2}, \%$ & $P_{Q}$ \\
\hline \multirow[t]{10}{*}{ PON1 (Q192R) } & $\mathrm{DN}$ & $563 / 608$ & Allele & $0.90(0.73-1.11)$ & 0.330 & 28.70 & 0.200 \\
\hline & & & Dominant & $0.84(0.49-1.15)$ & 0.530 & 60.50 & 0.052 \\
\hline & & & Homozygote & $0.79(0.41-1.53)$ & 0.490 & 61.10 & 0.040 \\
\hline & & & Heterozygote & $0.84(0.44-1.60)$ & 0.590 & 67.90 & 0.025 \\
\hline & & & Recessive & $0.91(0.64-1.31)$ & 0.630 & 42.70 & 0.095 \\
\hline & DR & $336 / 314$ & Allele & $0.87(0.56-1.33)$ & 0.510 & 69.00 & 0.045 \\
\hline & & & Dominant & $0.79(0.54-1.15)$ & 0.210 & 0.00 & 0.310 \\
\hline & & & Homozygote & $0.65(0.26-1.67)$ & 0.370 & 73.80 & 0.026 \\
\hline & & & Heterozygote & $0.84(0.56-1.27)$ & 0.410 & 0.00 & 0.760 \\
\hline & & & Recessive & $0.73(0.32-1.68)$ & 0.470 & 78.70 & 0.015 \\
\hline \multirow[t]{10}{*}{ PON1 (L55M) } & $\mathrm{DN}$ & $229 / 309$ & Allele & $1.03(0.79-1.34)$ & 0.820 & 0.00 & 0.540 \\
\hline & & & Dominant & $0.89(0.55-1.41)$ & 0.610 & 0.00 & 0.910 \\
\hline & & & Homozygote & $0.97(0.57-1.67)$ & 0.920 & 0.00 & 0.780 \\
\hline & & & Heterozygote & $0.83(0.49-1.39)$ & 0.470 & 0.00 & 0.950 \\
\hline & & & Recessive & $1.15(0.79-1.68)$ & 0.470 & 0.00 & 0.590 \\
\hline & DR & $392 / 537$ & Allele & $2.42(1.91-3.07)$ & $<0.001$ & 13.00 & 0.260 \\
\hline & & & Dominant & $5.76(3.14-10.55)$ & $<0.001$ & 0.00 & 0.810 \\
\hline & & & Homozygote & $10.53(5.59-19.86)$ & $<0.001$ & 0.00 & 0.740 \\
\hline & & & Heterozygote & $3.62(1.94-6.74)$ & $<0.001$ & 0.00 & 0.890 \\
\hline & & & Recessive & $3.56(2.61-4.86)$ & $<0.001$ & 0.00 & 0.520 \\
\hline \multirow[t]{10}{*}{ PON2 (S311C) } & $\mathrm{DN}$ & $408 / 492$ & Allele & $0.95(0.68-1.33)$ & 0.760 & 0.00 & 0.980 \\
\hline & & & Dominant & $0.72(0.52-1.01)$ & 0.055 & 0.00 & 0.690 \\
\hline & & & Homozygote & $0.73(0.53-1.02)$ & 0.069 & 0.00 & 0.800 \\
\hline & & & Heterozygote & $0.47(0.20-1.10)$ & 0.080 & 0.00 & 0.970 \\
\hline & & & Recessive & $0.89(0.68-1.18)$ & 0.430 & 2.50 & 0.450 \\
\hline & DR & $347 / 414$ & Allele & $1.14(0.84-1.55)$ & 0.390 & 42.70 & 0.190 \\
\hline & & & Dominant & $0.89(0.37-2.14)$ & 0.800 & 59.50 & 0.079 \\
\hline & & & Homozygote & $0.98(0.39-2.47)$ & 0.960 & 60.10 & 0.073 \\
\hline & & & Heterozygote & $0.81(0.33-1.98)$ & 0.640 & 57.80 & 0.091 \\
\hline & & & Recessive & $1.25(0.91-1.69)$ & 0.160 & 2.97 & 0.380 \\
\hline \multirow[t]{5}{*}{ PON2 (A148G) } & $\mathrm{DN}$ & $414 / 393$ & Allele & $0.77(0.51-1.16)$ & 0.210 & 0.00 & 0.920 \\
\hline & & & Dominant & $1.00(0.7-1.41)$ & 0.980 & 0.00 & 0.480 \\
\hline & & & Homozygote & $0.99(0.70-1.40)$ & 0.960 & 0.00 & 0.410 \\
\hline & & & Heterozygote & $0.78(0.22-2.75)$ & 0.700 & 0.00 & 0.930 \\
\hline & & & Recessive & $0.92(0.67-1.24)$ & 0.570 & 9.70 & 0.290 \\
\hline
\end{tabular}

PON, paraoxonase; DN, diabetic nephropathy; DR, diabetic retinopathy; OR, odds ratio; CI, confidence interval; $P_{Q}$, P-value for Q calculation.

association was found in any of the genetic models. The ORs for genetic effect were homogenous across the studies $\left(\mathrm{P}_{\mathrm{Q}}>0.1\right.$; $I^{2}=0 \%$; Table II).

Four studies were eligible for pooling of the genetic effects of L55M on DR. The allele model (Lvs., M) yielded a pooled OR of $2.42(95 \% \mathrm{CI}, 1.91-3.07)$ with mild heterogeneity $\left(\mathrm{P}_{\mathrm{Q}}=0.54\right.$; $I^{2}=13 \%$ ), indicating that the L allele was significantly higher in DR patients compared with that of DM controls. Similar or even more significant associations were also observed in the following genotype models: dominant (LL+LM vs., MM: $\mathrm{OR}=5.76$; 95\% CI, 3.14-10.55); homozygote (LL vs., MM: $\mathrm{OR}=10.53$; 95\% CI, 5.59-19.86); heterozygote (LM vs., MM:
$\mathrm{OR}=3.62 ; 95 \% \mathrm{CI}, 1.94-6.74)$; and recessive (LL vs., LM+MM: $\mathrm{OR}=3.56 ; 95 \% \mathrm{CI}, 2.61-4.86)$. The genotypic effects were homogenous, with $I^{2}$ values of $0 \%$ for the above-mentioned inherited models (Table II). Sensitivity analyses by excluding and including the study [Ergun et al (14)] that deviated from HWE yielded similar results, but was accompanied with moderate heterogeneity. No evidence of publication bias was identified.

Meta-analysis of PON2-S311C. Each of the three studies was performed to assess the association of S311C with DN and DR in Caucasian populations. The genetic effects were 
homogenous across DN studies $\left(I^{2}=0 \%\right)$, but mildly to moderately heterogeneous across studies in DR $\left(\mathrm{P}_{\mathrm{Q}}=0.073-0.38\right.$; $\left.I^{2}=3.0-60.1 \%\right)$. The pooled analysis showed no significant associations of S311C with DN or DR in any of the genetic models (Table II). No evidence of asymmetry was identified in the shape of the funnel plots (data not shown).

Meta-analysis of PON2-A148G. With regard to A148G, two studies exclusively assessed the association of A148G with DN only. The analysis showed no significant association between A148G and DN in any of the following genetic models: allele (A vs., G: OR=0.77; 95\% CI, 0.51-1.16), dominant model (AA+AG vs., GG: $\mathrm{OR}=1.00 ; 95 \% \mathrm{CI}$, 0.70-1.41), homozygote model (AA vs., GG: OR=0.99; 95\% CI, 0.70-1.40), heterozygote (AG vs., GG: $\mathrm{OR}=0.78$; 95\% CI, 0.22-2.75), and recessive model (AA vs., AG+GG: $\mathrm{OR}=0.92 ; 95 \% \mathrm{CI}, 0.67-1.24)$. The ORs for all genetic effects were homogenous across studies $\left(I^{2}=0 \%\right)$, with the exception of mildly heterogeneous in the recessive model $\left(\mathrm{P}_{\mathrm{Q}}=0.29\right.$; $I^{2}=9.7 \%$; Table II).

\section{Discussion}

In the present study, a systematic review and meta-analysis was performed to examine the associations of four well-evaluated polymorphisms in $P O N$ with DN and DR. The results indicated that the PON1-L55M polymorphism was significantly associated with DR, which remained following sensitivity analyses. The observations were consistent with a majority of the previous studies investigated. The conflicting results obtained on a Turkish population by Ergun et al (14) may be due to differences in diabetes control selection and statistical power, as well as ethnical background (14). Genetic effect of $\mathrm{L}$ allele yielded a higher risk of having DR (between 3.56and 10.53-fold in various genetic models), indicating that it is worthy of in-depth analysis, particularly its biological functions. However, such an association was not detected in DN, which may be due to the limited studies, various phenotypes and heterogeneity in the genetic susceptibility between DN and DR. Further examination in larger cohorts are therefore required. Nevertheless, the present study highlighted results for the genetic association of functional variant L55M and DR, which is definitely likely to lead to increased research interest, particularly for its biological effect.

As aforementioned, low-density lipoprotein (LDL) oxidation is key for the development of microvascular diseases (21). PON activity affects the efficiency of HDL on the inhibition of LDL oxidation (22). Moreover, lower PON activity has been examined in type 2 diabetes patients, which has been implicated in the development of diabetic microvascular complications (23). The PON1-L55M polymorphism has been found to modify the serum concentration and enzyme activity of PON $(24,25)$. Thus, these circumstantial and laboratory results suggest a critical role for L55M in the development of DR, although, the exact molecular mechanisms remain elusive.

In the current study, no evident associations were found in the remaining three variants (PON1-Q192R, PON2-S311C and PON2-A148G) with DN or DR under any of the genetic models. Therefore, the results suggest that these polymorphisms may not be associated with diabetic microvascular complications, particularly for DN and DR.

The present study had a number of strengths. Firstly, to the best of our knowledge, this is the first meta-analysis to investigate the associations of $P O N$ gene polymorphisms with DN and DR. Secondly, the methods of the meta-analysis were carefully designed; explicit search strategy based on computer-assisted and manual search methods allowed almost all relevant studies to be included and the conclusions are based on conservative estimations. However, specific limitations also existed; the number of available studies is not sufficient enough for every variant in the meta-analysis, particularly for specific subgroups. Thus, certain analyses based on $<2$ studies may not be powered to detect modest association and must be assessed cautiously. Additional studies of larger sample sizes and containing more detailed information are required. An additional potential drawback is that the majority of studies were clinic-based resources, which may produce overestimated genetic effects. However, this is unlikely to be significant in the present study, which considered the significantly statistical power, together with its biological relevance.

In conclusion, the current meta-analysis highlighted conclusive results for the robust association between PON1-L55M polymorphisms with DR. The results also demonstrated that the remaining three variants (PON1-Q192R, PON2-S311C and $P O N 2-\mathrm{A} 148 \mathrm{G})$ may not be associated with DN or DR. Larger association studies and functional analyses of PON1 are required to elucidate the pathological mechanisms of the diabetic microvascular complications.

\section{Acknowledgements}

The authors would like to thank Dr Liu Guodong from the Department of Endocrinology (Harbin Medical University, Harbin, China) and anonymous reviewers for their useful comments and language editing, which have greatly improved the manuscript.

\section{References}

1. Resnikoff S, Pascolini D, Etya'ale D, et al: Global data on visual impairment in the year 2002. Bull World Health Organ 82: 844-851, 2004

2. Sheetz MJ and King GL: Molecular understanding of hyperglycemia's adverse effects for diabetic complications. JAMA 288: 2579-2588, 2002.

3. Stratton IM, Kohner EM, Aldington SJ, et al: UKPDS 50: risk factors for incidence and progression of retinopathy in Type II diabetes over 6 years from diagnosis. Diabetologia 44: 156-163, 2001.

4. Pettitt DJ, Saad MF, Bennett PH, Nelson RG and Knowler WC: Familial predisposition to renal disease in two generations of Pima Indians with type 2 (non-insulin-dependent) diabetes mellitus. Diabetologia 33: 438-443, 1990.

5. The Diabetes Control and Complications Trial Research Group: Clustering of long-term complications in families with diabetes in the diabetes control and complications trial. Diabetes 46: 1829-1839, 1997.

6. Canani LH, Araki S, Warram JH and Krolewski AS: Comment - to: Pinizzotto M, Castillo E, Fiaux M, Temler E, Gaillard RC, Ruiz J (2001) Paraoxonase 2 polymorphisms are associated with diabetic nephropathy in Type II diabetes. Diabetologia 44: 104-107. Diabetologia 44: 1062-1064, 2001.

7. Kao Y, Donaghue KC, Chan A, Bennetts BH, Knight J and Silink M: Paraoxonase gene cluster is a genetic marker for early microvascular complications in type 1 diabetes. Diabet Med 19: 212-215, 2002. 
8. Pinizzotto M, Castillo E, Fiaux M, Temler E, Gaillard RC and Ruiz J: Paraoxonase2 polymorphisms are associated with nephropathy in Type II diabetes. Diabetologia 44: 104-107, 2001.

9. Lyons TJ, Li W, Wells-Knecht MC and Jokl R: Toxicity of mildly modified low-density lipoproteins to cultured retinal capillary endothelial cells and pericytes. Diabetes 43: 1090-1095, 1994.

10. Morrison AC, Bare LA, Luke MM, et al: Single nucleotide polymorphisms associated with coronary heart disease predict incident ischemic stroke in the atherosclerosis risk in communities study. Cerebrovasc Dis 26: 420-424, 2008.

11. Luke MM, Lalouschek W, Rowland CM, et al: Polymorphisms associated with both noncardioembolic stroke and coronary heart disease: vienna stroke registry. Cerebrovasc Dis 28: 499-504, 2009.

12. Durrington PN, Mackness B and Mackness MI: Paraoxonase and atherosclerosis. Arterioscler Thromb Vasc Biol 21: 473-480, 2001.

13. Araki S, Makita Y, Canani L, Ng D, Warram JH and Krolewski AS: Polymorphisms of human paraoxonase 1 gene (PON1) and susceptibility to diabetic nephropathy in type I diabetes mellitus. Diabetologia 43: 1540-1543, 2000.

14. Ergun MA, Yurtcu E, Demirci H, et al: PON1 55 and 192 gene polymorphisms in type 2 diabetes mellitus patients in a Turkish population. Biochem Genet 49: 1-8, 2011.

15. Kao YL, Donaghue K, Chan A, Knight J and Silink M: A variant of paraoxonase (PON1) gene is associated with diabetic retinopathy in IDDM. J Clin Endocrinol Metab 83: 2589-2592, 1998

16. Kordonouri O, James RW, Bennetts B, et al: Modulation by blood glucose levels of activity and concentration of paraoxonase in young patients with type 1 diabetes mellitus. Metabolism 50: 657-660, 2001.

17. Mackness B, McElduff $\mathrm{P}$ and Mackness MI: The paraoxonase-2-310 polymorphism is associated with the presence of microvascular complications in diabetes mellitus. J Intern Med 258: 363-368, 2005
18. Murata M, Maruyama T, Suzuki Y, Saruta T and Ikeda Y: Paraoxonase $1 \mathrm{Gln} / \mathrm{Arg}$ polymorphism is associated with the risk of microangiopathy in Type 2 diabetes mellitus. Diabet Med 21: 837-844, 2004

19. Tiwari AK, Prasad P, B KT, et al: Oxidative stress pathway genes and chronic renal insufficiency in Asian Indians with Type 2 diabetes. J Diabetes Complications 23: 102-111, 2009.

20. Fleiss JL: The statistical basis of meta-analysis. Stat Methods Med Res 2: 121-145, 1993.

21. Nivoit P, Wiernsperger N, Moulin P, Lagarde M and Renaudin C: Effect of glycated LDL on microvascular tone in mice: a comparative study with LDL modified in vitro or isolated from diabetic patients. Diabetologia 46: 1550-1558, 2003.

22. Mackness B, Durrington PN, Boulton AJ, Hine D and Mackness MI: Serum paraoxonase activity in patients with type 1 diabetes compared to healthy controls. Eur J Clin Invest 32: 259-264, 2002

23. Tsuzura S, Ikeda Y, Suehiro T, et al: Correlation of plasma oxidized low-density lipoprotein levels to vascular complications and human serum paraoxonase in patients with type 2 diabetes. Metabolism 53: 297-302, 2004.

24. Humbert R, Adler DA, Disteche CM, Hassett C, Omiecinski CJ and Furlong CE: The molecular basis of the human serum paraoxonase activity polymorphism. Nat Genet 3: 73-76, 1993.

25. Garin MC, James RW, Dussoix P, et al: Paraoxonase polymorphism Met-Leu54 is associated with modified serum concentrations of the enzyme. A possible link between the paraoxonase gene and increased risk of cardiovascular disease in diabetes. J Clin Invest 99: 62-66, 1997. 